\title{
Zinc in relation to type 1 and type 2 diabetes: An overview
}

\author{
U. Singh \\ Department of Food and Nutrition, Institute of Home Science, Bundelkhand University, Jhansi- 284128, (U.P), INDIA \\ E-mail: usuttarasingh@gmail.com
}

Received: February 06, 2014; Revised received: October 05, 2014; Accepted: December 10, 2014

\begin{abstract}
In type 1 diabetes, there is a lack of insulin production and in type 2, diabetes resistances to the effects of insulin are predominant. Both type 1 and type 2 have the same long-term complications. Diabetes effects zinc homeostasis in many ways, although it is most probably the hyperglycemia which is responsible for the increased urinary loss and decreases in total body zinc. The role of $\mathrm{Zn}$ deficiency exacerbates the cytokine-induced damage in the autoimmune attack which destroys the islet cell in type 1 diabetes, is unclear. Since $\mathrm{Zn}$ plays a clear role in the synthesis, storage and secretion of insulin as well as conformational integrity of insulin in the hexameric form, the decreased $\mathrm{Zn}$, which affects the ability of the islet cell to produce and secrete insulin in type 2 diabetes. Oxidative stress and cellular dysfunction in diabetes may be related to increased intracellular oxidants and free radicals associated with decrease in intracellular $\mathrm{Zn}$ and in $\mathrm{Zn}$ dependent antioxidant enzymes. There appears to be a complex interrelationship between $\mathrm{Zn}$ and both type 1 and type 2 diabetes. $\mathrm{Zn}$ plays a key role in the cellular antioxidative defense. Dysfunctional zinc signaling is associated with a number of chronic disease states including cancer, cardiovascular disease, Alzheimer's disease, and diabetes. Cellular homeostasis requires mechanisms that tightly control the uptake, storage, and distribution of zinc. This is achieved through the coordinated actions of zinc transporters and metallothioneins.
\end{abstract}

Keywords: Antioxidants, Homeostasis, Hyperglycemia, Juvenile, Zinc

\section{INTRODUCTION}

It is not always clear which comes first: the effects of diabetes mellitus and hyperglycemia on zinc metabolism or the effects that follow alterations in $\mathrm{Zn}$ homeostasis on carbohydrate metabolism. There are several forms of disordered glucose metabolism which are referred to collectively as diabetes. While all diabetes mellitus syndromes have in common some degree of hyperglycemia, the hyperglycemia is a symptom, not the metabolic error itself (Brange and Langkjoer, 1993). Zinc is an essential trace element that is indispensable for its role in maintaining normal physiological function and cellular homeostasis (Little et al., 2010). Type I diabetes mellitus has been called "juvenile" diabetes since it most often occurs in children and young adults, but it is clear that an individual of any age can develop this metabolic error. The current nomenclature for this form of diabetes is "insulin dependent diabetes mellitus (IDDM), type I. This form of energy generation results in the production of ketone bodies and organic acids, primarily acetoacetic and beta hydroxybutyric acids, with the consequence of the development of severe metabolic acidosis. This results in the typical picture of diabetic ketoacidosis (DKA). These patients are dependent on insulin for life, not just control of the blood glucose level. In general, a dose sufficient to replace the normal production of insulin will result in acceptable normalization of the blood glucose. This is approximately 20 to 25 units per day.

The metabolic errors in type II diabetes are markedly different from those observed in type I diabetes. This disorder has been referred to as "adult" onset of diabetes. Type II may be seen in children and young adults, although it is uncommon. This was seen often enough, however, to have been given a name of its own-"Maturity onset diabetes of youth (MODY)" -syndrome standing for. No matter the age, in type II diabetes the pancreatic islet cells are capable of making large quantities of insulin, at least at the beginning of the disease (Arquilla et al., 1978). In the healthy normal individual, insulin binds to a cell membrane receptor and, through several pathways, results in the transport of glucose across the membrane. The intracellular events associated with the activation of glucose transport after the signal from the insulin -receptor complex is received are called the "post -receptor" events. To a great extent, it is the failure of the post-receptor events that results in hyperglycemia in and of itself. In response to the hyperglycemia, the pancreatic islets produce greater and greater quantities of insulin which results in a "down regulation" of the number of insulin receptors on the cell membrane, which compounds the problem (Arthur and Chausmer 1998). This results in both hyperglycemia and hyperinsulinemia. Additionally, the beta cells cannot make enough 
insulin to normalize the glucose, suggesting an error in the ability of the beta cell to synthesize insulin. As the disease progresses, there may be an exhaustion of the beta cells with a relative inability to keep up with the needs for insulin production. The resultant decrease in insulin produced results in even greater hyperglycemia. In type II diabetes there is plenty of insulin, it just doesn't work very well, and the islets cannot produce enough insulin to compensate. There is no intracellular starvation and no ketoacidosis. In type II diabetes, oral medications can improve the cellular sensitivity to insulin and increase insulin release. If an adequate response cannot be obtained with these compounds, exogenous insulin may be required to normalize the blood glucose, but these patients are not dependent on insulin for existence as are the type I diabetics. Since these patients are resistant to insulin, the daily dose of insulin necessary to approximate normalization of the blood glucose is significantly higher than the average daily production. In these patients, therapeutic doses of insulin in addition to the endogenously produced insulin generally range from 50 to 200 units per day as compared to the 20 to 30 units per day produced by a normal individual (Levine et al., 1983). Pregnancy may frequently be associated with disorders of glucose metabolism resulting in gestational diabetes. Since both hyperglycemia and hyperinsulinemia are detrimental to the fetus, very close control of the blood glucose during pregnancy is required. Women who have had gestational diabetes may go on to become overtly diabetic after pregnancy, may never have any problem with glucose metabolism other than when pregnant, or may be "normal" for many years before developing diabetic symptoms. Because insulin is a powerful growth factor and can be produced by the fetal pancreas in response to high levels of maternal blood glucose, prolonged maternal hyperglycemia can result in macrosomia, or large/heavy for gestational age, babies, congenital fetal abnormalities and spontaneous abortion/miscarriage. Because of the serious complications associated with both the hyperglycemic and hyperinsulinemic states, the diagnostic criteria for gestational diabetes are considerably more stringent than for other forms of diabetes. A lesser degree of hyperglycemia is necessary for the diagnosis of gestational diabetes and the level of control of the hyperglycemia with exogenous insulin during pregnancy is much -"tighter"'- (Raz et al., 1988).

\section{ZINC AND INSULIN INTERACTIONS}

Insulin is secreted by the beta cell both tonically (at a constant low level release rate) and as a high level spike in response to an immediate glucose load such as a meal. Long before there was any biochemical evidence for the relationship between zinc and insulin in the beta cell, it was clear that the addition of zinc to insulin would change the time course of the effect of a given dose of insulin. Insulin is produced by the beta cell of the pancreatic islets as a single chain peptide that is bent around itself and linked by two inter-chain disulfide bonds. This proinsulin is cleaved by the removal of an intracellular chain fragment known as the "C-peptide" to form two peptide chain (alpha and beta) molecules of 51 amino acids cross-linked to each other by inter-chain disulfide bonds. This is the insulin monomer. In the presence of zinc within the cell, insulin monomers assemble to a dimeric form for storage and secretion as the zinc crystal (Arthur and Chausmer 1998). Zalewski (1994) reported that high concentrations of glucose and other secretagogues decrease the islet cell labile $\mathrm{Zn}$ and video fluorescence analysis showed $\mathrm{Zn}$ concentrated in the islet cells was related to the synthesis, storage and secretion of insulin (Zalewski et al., 1994).

In vitro, in the presence of zinc and at neutral $\mathrm{pH}$, dimeric insulin assembles further into a hexamer consisting of three dimeric units. This form of insulin is relatively stable and it is this hexameric crystal which is the commonly used pharmacologic form. Changes in the tertiary conformation of the hexamer may also result in significant biologically important changes relating to release rate and consequent biologic activity (Brader and Dunn, 1991; Brange and Langkjoer, 1993). In vitro data suggest that insulin binds to isolated liver membranes to a greater extent and that there is less degradation when $\mathrm{Zn}$ is co-administered with insulin (Arquilla et al., 1978).

Zinc has three major biological roles: catalytic, structural and regulatory (Chasapis et al., 2011). The catalytic and structural role of zinc is well recognized, and there are many noteworthy reviews on these functions (Maret , 2011 and $\mathrm{Lu}$ and $\mathrm{Fu}$ 2007) and references therein). For example, zinc is a structural constituent in numerous proteins, including growth factors, cytokines, receptors, enzymes, and transcription factors belonging to cellular signaling pathways (Fukada et al., 2011). Moreover, it is implicated in numerous cellular processes as a cofactor for an estimated 3000 human proteins including enzymes, nuclear factors, and hormones (Andreini et al., 2006). Therapeutic interventions to alter the zinc insulin interactions have also been attempted. With the development of genetic - "engineering,"- it has become possible to develop analogues of insulin which do not form $\mathrm{Zn}$ insulin hexamers which result in a more rapid absorption from the injection site (Garg et al., 1994).

\section{EFFECTS OF DIABETES ON ZINC METABOLISM}

The predominant effect on zinc homeostasis of diabetes is hypozincemia which may be the result of hyperzincuria or decreased gastrointestinal absorption of $\mathrm{Zn}$ or both. It appears that the hyperzincuria, at least, is a result more of hyperglycemia than any specific effect of endogenous 
or exogenous insulin on the renal tubule. In 20 age/sex matched controls and 30 diabetic patients, the serum $\mathrm{Zn}$ was approximately $40 \%$ lower in the diabetes group. No differences within the diabetes group were observed when treatment differences between insulin, oral hypoglycemic agent or combination or between type I and type II diabetes were evaluated (Garg et al., 1994). Isbir et al. (1994) demonstrated a $20 \%$ decrease in serum in type I diabetes, apparently the result of hyperzincuria (Isbir et al., 1994). El Yazigi et al (1993) evaluated both type I and II diabetics and found the absolute and creatinine corrected excretions were greater in diabetics than in matched controls and found a positive correlation between $\mathrm{Zn}$ excretion and hemoglobin Alc concentrations. Study suggests that insulin treatment of diabetes may reduce the hyperzincuria while oral agents had no effect on the increased zinc excretion seen in type II diabetes (Honnorat et al., 1992). These data suggest hyperglycemia as the basis for the hyperzincuria, but since type I and type II patients were taking insulin or oral hypoglycemic agents which increase insulin secretion, an effect of insulin itself has not been eliminated. There is loss of a large amount of zinc from the body via the urine. The source of the zinc that is excreted remains incompletely resolved. There is a concurrent hypozincemia and a decrease in tissue zinc stores, but it is not clear if this is a result of the hyperzincuria or an independent event related to the effect of insulin or hyperglycemia on loss of $\mathrm{Zn}$ from the tissue stores with a resultant loss of zinc to the plasma from where it is excreted with a net loss of total body zinc and eventual hypozincemia. It has been postulated that hyperglycemia interferes with the active transport of $\mathrm{Zn}$ back into the renal tubular cells. In dogs, at least, experimentally induced hyperglycemia resulted in significant hyperzincuria (Kinlaw et al., 1983). In rats made diabetic by streptozo-tocin (STZ), who therefore do not have any genetic code for the metabolic defects seen in diabetes, increased zinc excretion has been routinely observed. Administration of insulin with reduction in the hyperglycemia reduces, but does not appear to completely ameliorate the hyperzincuria (Lau and Failla, 1984; Failla and Gardil, 1985).

Alc and urinary $\mathrm{Zn}$ excretion. Hemoglobin Alc is a reliable quantitative indicator of long-term hyperglycemia. In a study of 175 patients with both Type I and II diabetes, there was a positive correlation between urinary zinc excretion and hemoglobin Alc (El-Yazigi et al., 1983). In a group of age/sex matched diabetic and normal subjects, cellular loss of zinc was documented from granulocytes as a marker for intracellular zinc status for the body in general in otherwise healthy individuals. Plasma $\mathrm{Zn}$ was $17 \%$ lower in the diabetic group than the control group but intra-cellular $\mathrm{Zn}$ differences did not reach statistical significance suggesting that the renal effects may be predominant in the eventual production of total body $\mathrm{Zn}$ depletion in diabetes (Williams et al., 1995). While hyperzincuria is almost uniformly found in diabetes, decreases in tissue. McNair et al., (1981) confirmed hyperzincuria in relationship to the degree of hyperglycemia, but not glycosuria. These studies, however, have not demonstrated hypozincemia in the presence of reproducible hyperzincuria and the authors suggest that tissue and plasma zinc losses via the urine may be ameliorated by a compensatory increase in gastrointestinal absorption of zinc. Other data, however, have suggested that there is also a defect in zinc absorption associated with hyperglycemia or diabetes. Kinlaw et al., (1983) demonstrated abnormal $\mathrm{Zn}$ tolerance tests in diabetic patients suggestive of decreased absorption. Escobar et al., (1995) also demonstrated a down regulation of fractional $\mathrm{Zn}$ transport which may be related to increased production of metallothionein in diabetics. Metallothionein is an intracellular cation binding protein which appears to act as, among other things, an inhibitor of $\mathrm{Zn}$ transport. This decrease in gastrointestinal absorption, coupled with hyperzincuria, could account for significant loss of intracellular $\mathrm{Zn}$.

The effects of hyperinsulinism/hyperglycemia on tissue concentrations are difficult to evaluate. Cordova (1994) found increased tissue $\mathrm{Zn}$ in liver, muscle and kidney in strep- tozotocin diabetic rats. These are the tissues that are responsive to insulin in terms of glucose transport. When the rats were exercised, which allows insulin independent glucose transport in muscle, tissue $\mathrm{Zn}$ decreased suggesting zinc may be lost from cells as glucose is translocated into muscle. Other studies also suggest that these same tissues increase zinc concentrations in the diabetic state (Levine et al., 1983; Failla and Gardil, 1985). Conflicting data was demonstrated in another approach to the tissue/zinc question in the spontaneous diabetic sand rat animal model. Unlike the STZ diabetes model in which there is no genetic component, these rats may have a different mechanism for cellular responses to insulin and hyperglycemia. In these animals, hyperglycemia/hyperinsulinemia was associated with reduced tissue zinc in liver, kidney and muscle. Again, it should be noted that these are tissues in which insulin affects glucose metabolism. There were no changes in bone zinc (Raz et al., 1988). Serum and urinary zinc levels were evaluated in patients with diabetes, congestive heart failure and both states. No differences in serum zinc levels were observed in any group, but hyperzincuria was greater in the group with both states than with either diabetes or congestive heart failure alone. Renal function, as evaluated by serum creatinine, was not different between the groups (Golik et al., 1993).

It is clear that urinary excretion of $\mathrm{Zn}$ is markedly increased in individuals with diabetes, if hyperglycemia is the primary etiology, replacement with oral $\mathrm{Zn}$ supplementation should provide sufficient treatment. High dose supplementation in Type I diabetics and 
normal individuals was evaluated by serum and urine zinc and mononuclear cell $\mathrm{Zn}$ concentrations. Zinc excretion and mononuclear zinc concentrations increased by a similar amount in both groups (Cunningham et al., 1994).

\section{EFFECTS OF ZINC ON DIABETES MELLITUS (PRIMARY DISEASE EFFECTS)}

Quarterman et al., (1966) demonstrated that diet induced zinc deficiency in rats resulted in a decrease in the ability of the pancreas to secrete insulin in response to a glucose load a hallmark of diabetes. Boquist et al., (1968) demonstrated a decrease in glucose tolerance with no change in insulin production in zinc deficient hamsters in response to an increase glucose load. This suggested at least two roles for zinc: an inhibition of the post insulin receptor intracellular events which results in a decreased glucose tolerance, and a relative decrease in insulin secretion, since the elevated glucose should produce a more exuberant insulin response. This suggests that zinc deficiency also reduced the ability of the pancreas to respond appropriately. Decreased granulation of the islet cell with both light and electron microscopy in zinc deficiency. Since zinc is intrinsic to the storage granulization of insulin within the beta cell and increased insulin secretion reduces beta cell zinc concentration, these data are compatible with decreased islet cell insulin content in zinc deficiency states (Engelbart and Kief, 1970).

The etiology of Type I diabetes is the result of autoimmune attack on the beta cell with subsequent destruction of the cell. One proposed mechanism by which immune processes damage cells is through cytokine mediated induction of intracellular oxidizing agents, particularly free radicals. A similar mechanism is involved in the cell damage that occurs in the tissues associated with the "complications" of diabetes, particularly the retina in retinopathy. These oxidizing agents include free radicals, which have unpaired electrons, such as superoxide radicals, alkoxyl and peroxyl radicals, hydrogen peroxide, and lipid peroxides. Because zinc is a necessary factor in a variety of "antioxidant" enzymes, particularly superoxide dismutase, catalase and peroxidase, al- terations of zinc metabolism such that adequate zinc is unavailable for these enzymes might be expected to contribute to the tissue damage observed in diabetes (Sumovski et al., 1992).

Cytokines are one of the mediators of the immune response; they are potential candidates as the mediators of cell destruction in the autoimmune attack on the human beta cell which occurs in type I diabetes. Rabinovitch et al. (1996) examined the relationship between cytokine induced (interleukin $1 \mathrm{~b}$, tumor necrotic factor (TNF) and interferon gamma) pancreatic beta cell destruction, production of malondialdehyde (MDA), an end product of lipid peroxidation; and nitrite, the end product of nitric oxide. These studies suggested cytokines are toxic to the human beta cell by producing oxygen free radicals, lipid peroxidation, and aldehyde production in the islets and that MDA was one of the cytotoxic mediators (Sumovski et al., 1992 and Rabinovitch et al., 1996).

$\mathrm{Zn}$ metallothionein complex in the islet cell provides protection against free radicals produced in the cell from any cause, and certainly the immune mediated cytokine provoked oxidative stress would be a significant oxidative stress. The more depleted the intracellular $\mathrm{Zn}$ stores, the less able the cell is to defend itself against this oxidative load. This provides a potential mechanism for zinc deficiency to affect the progress of type I diabetes. There is also some data suggesting that the same mechanism of intracellular oxidative load may be responsible for the streptozotocin induced experimental diabetes. Zimny et al., (1993) have demonstrated induction of metallothionein production in the islet cell in response to STZ induced $-\mathrm{OH}$ radical production. Yang and Cherian (1994) demonstrated STZ-induced lipid peroxidation and decreased superoxide -dismutase (SOD). The lipid peroxidation was mitigated by pretreatment with intraperitoneal $\mathrm{Zn}$ to induce increased production of metallothionein, although no changes in SOD were noted. Metallothionein requires $\mathrm{Zn}$ for activation and a paucity of $\mathrm{Zn}$ would exacerbate the deleterious effects of STZ on the islet cell. Roza et al. (1995) reported marked decreases in pancreatic SOD and catalase antioxidant activity which preceded the loss of beta cell function. All of these data suggest a role for $\mathrm{Zn}$ in the protection of the beta cell against the immune mediated free radical attack, but it is not clear if this related to the genetic factors in the predisposition to diabetes or of the islets itself prior to cell destruction.

In type II diabetes there is no good evidence for oxidative stress as a major factor in the development of either insulin deficiency or islet cell damage, but there is clear evidence for increased secretion of insulin, at least early in the progress of the disease. Since Zn leaves the cell with insulin, the greater secretion of insulin causes cellular depletion of $\mathrm{Zn}$. The cell can make more insulin, but it cannot make more $\mathrm{Zn}$ and, with hyperzincuria, the $\mathrm{Zn}$ co-secreted is more likely to be excreted and not available for re-uptake into the cellular pool. With the slow loss of intracellular zinc, the less insulin is secreted for a given glucose level and the islet cell becomes more vulnerable to all sorts of damage. This matches the clinical picture in which, after prolonged hyperglycemia and inability of the islet cell to make enough insulin to control the glucose, there is a loss of islet cell altogether. This provides a mechanism by which $\mathrm{Zn}$ deficiency may affect the progress of type II diabetes (Spreitsma and Schuitemaker, 1994). 


\section{EFFECTS OF ZINC ON DIABETES MELLITUS (SECONDARY COMPLICATIONS)}

While diabetes, particularly type I, as a primary event is devastating to the body, it is the long-term complications which provide the bulk of the economic and social costs Hyperglycemia is a major culprit in the development of the microvascular complications which include retinopathy, nephropathy, neuropathy and the small vessel occlusions as well as birth defects including fetal malformations and macrosomia. In mice made diabetic with STZ there was a marked increase in fetal loss and malformation as compared with nondiabetic mice. In similar, but transgenic mice for the human copper zinc superoxide dismutase $(\mathrm{Cu}-\mathrm{Zn}$ SOD), there were fewer fetal malformations than in controls without the gene suggesting SOD reduces diabetic embryopathy, presumably by reducing oxygen free radicals (Hagay et al., 1995).

\section{Conclusion}

Diabetes of any type can be a devastating personal health problem and is certainly a very significant public health problem. It would be nice if there were some relatively simple interventions which would help ameliorate the damage done by this disease, either as the primary problem or by its hallmark physiologic signature, hyperglycemia.

The ubiquitous nature of zinc in physiological systems suggests that atypical levels are likely to have many biological and clinical effects. The true significance of zinc in cellular signaling is just emerging. In this context the zinc transporters play an essential role in insulin and glucose homeostasis. The inhibition of protein tyrosine phosphatases by zinc under physiological conditions involving zinc transporter mechanisms has widespread implications for understanding insulin resistance and disease progression.

\section{REFERENCES}

Andreini, C., Banci, L., Bertini, I. and Rosato, A. (2006). Counting the Zinc-proteins encoded in the human genome. Journal of Proteome Research, vol. 5, no. 1, 196-201.

Arthur, B. and Chausmer (1998). Zinc, Insulin and Diabetes. Journal of the American College of Nutrition, 17, 2: 109 -115 .

Arquilla, E.R. Packer, S. Tarmas, W. and Miyamoto, S. (1978). The effect of zinc on insulin metabolism. Endocrinology, 103: 1330-1449.

Arquilla, E.R. Thiene, P. Brugman, T. Ruess, T. and Sugiyama, R. (1978). Effects of zinc ion on the conformation of antigenic determinants on insulin. Biochem. J., 175: $289-297$.

Brader, M.L. and Dunn, M.F. (1991). Insulin hexamers: new conformations and applications. Trends Biochem. Sci., 16: 341-345.

Brange, J. and Langkjoer, L. (1993). Insulin structure and stability. Pharm. Biotechnol.,5: 315-350.

Cordova, A. (1999). Zn content in selected tissues in streptozotocin diabetic rats after maximal exercise. Biol. Trace El.
Res., 42: 209-215.

Cunningham. J, Fu, A. Mearkle, P. and Brown, R. (1994). Hyperzincuria in individuals with insulin dependent diabetes mellitus: concurrent zinc status and the effect of high dose zinc supplementation. Metabolism, 43: $1558-1562$.

Chasapis, C., Loutsidou, A., Spiliopoulou, C. and Stefan- idou, M. (2011). Zinc and human health: an update. Archives of Toxicology, vol. 86, 1-14.

El-Yazigi, A. Hannan, N. and Raines, D. (1993). Effect of diabetic state and related disorders on the urinary excretion of magnesium and zinc in patients. Diabetes Res., 22: 67-75.

Engelbart, K. and Kief, H. (1970). The functional behaviour of zinc and insulin contain in the pancreatic islet cells of rats. Virchows Archives, Cell Pathol., 4: 294-302.

Escobar, O. Sandoval, M. Vargas, A. and Hempe, J. (1995). Role of metallothi- oneien and cysteine rich intestinal protein in the regulation of $\mathrm{Zn}$ absorption by diabetic rats. Ped. Res., 37: 321-327.

Failla, M.L. and Gardil, C. (1985). Influence of spontaneous diabetes on tissue status of zinc, copper, and manganese in BB Wistar rats. PSEBM., 180: 317-322.

Garg, V. Gupta, R. and Goal, R. (1994). Hypozincemia in diabetes mellitus. J.A.P.I., 42: 720-721.

Golik, A. Cohen, N. Ramot, Y. Maor, J. Moses, R. Weissgarten, J. Leonov, Y. and Modai, P. (1993). Type II diabetes mellitus, congestive heart failure and zinc metabolism. Biol. Trace El. Res., 39: 171-175.

Fukada, T., Yamasaki, S., Nishida, K., Murakami, M. and Hirano, T. (2011). Zinc homeostasis and signaling in health and diseases-Zinc signaling. Journal of Biological Inorganic Chemistry, vol. 16, 1123-1134.

Hagay, Z. Weiss, Y. Zusman, I. Peled-Kamar, M. Reese, E. Erikson, U. and Groner, Y. (1995). Prevention of diabetes associated embryopathy by overexpression of the free radical scavenger copper zinc superoxide dismutase in transgenic mouse embryos. Am. J. Obstet. Gynecol., 173: 1036-1041.

Honnorat, J. Accominotti, M. Broussolle, C. Fleuret, A. Vallon, J. and Orgiazzi, J. (1992). Effects of diabetes type and treatment on zinc status in diabetes mellitus. Biol. Trace Elem. Res., 32: 311-316.

Isbir, T. Tamer, A. Taylor, A. and Isbir, M. (1994). Zinc, copper and magnesium status in insulin dependent diabetes. Diabetes Res., 26: 41-45.

Kinlaw, W.B. Levine, S. Morley, J. Silvis, S. and McClain, C. (1983). Abnormal $\mathrm{Zn}$ metabolism in type II diabetes mellitus. Am. J. Med., 75: 273-277.

Lau, A. and Failla, M. (1984). Urinary excretion of zinc, copper and iron in streptozotocin diabetic rat. J. Nutr., 114: 224.

Levine, A.S. McClain, C. Handwerger, B. Brown, D. and Morley, J. (1983). Tissue $\mathrm{Zn}$ status of genetically diabetic and streptozotocin induced dia- betic mice. Am. J. Clin. Nutr., 37: 382-386.

Little, P.J., Bhattacharya, R., Moreyra, A.E. and Korich- neva, I. L. (2010). Zinc and cardiovascular disease. Nutrition, vol. 26, no. 11-12, 1050-1057.

$\mathrm{Lu}, \mathrm{M}$. and Fu, D. (2007). Structure of the Zinc transporter YiiP. Science, vol. 317, no. 5845, 1746-1748.

Maret, W. (2011). Metals on the move: Zinc ions in cellular regulation and in the coordination dynamics of Zinc proteins. BioMetals, vol. 24, no. 3, 411-418.

Maret, W. (2011). New perspectives of Zinc coordination 
environments in proteins. Journal of Inorganic Biochemistry, 111: 110-116.

McNair, P. Kiilerich, S. Christiansen, C. Christiansen, M. Madsbad, S. and Transbol, I. (1981).Hyperzincuria in insulin treated diabetes mellitus-its relation to glucose homeostasis and insulin administration. Clinica. Chimica. Acta., 112: 343-348.

Quarterman, J. Mills, C. and Humphries, W. (1966). The reduced secretion of and sensitivity to insulin in $\mathrm{Zn}$ deficient rats. BBRC., 25: 354-358.

Rabinovitch, A. Suarez-Pinzon, W. Strynadka, K. Lakey, J. and Rajotte, R. (1996). Human pancreatic islet beta cell destruction by cytokines in- volves oxygen free radicals and aldehyde production. J. Clin. Endocrinol. Metab., 81: 3197-3202.

Raz, I. Adler, J.H. and Havivi, E. (1988). Altered tissue content of trace metals in diabetic hyperinsulinemic sand rats. Diabetologia, 31: 329-333.

Roza, A. Pieper, G. Johnson, C. and Adams, M. (1995). Pancreatic antioxidant enzyme activity in normoglycemic diabetic prone BB rats. Pancreas, 10: 53-58.

Spreitsma, J. and Schuitemaker, G. (1994). Diabetes can be prevented by reducing insulin production. Medical Hypotheses, 42: 15-23.

Sumovski, W. Baquerizo, H. and Rabinovich, A. (1992). Oxygen free radical scavenger protect rat islet cells from damage by cytokines. Diabetologica, 32: 792-796.

Williams, N.R. Rajput-Williams, J. West, J. Nigdikar, S. Foote, J. and Howard, A. (1995). Plasma, granulocyte and mononuclear cell copper and zinc in patients with diabetes mellitus. Analyst, 120: 887-890.

Yang, J. and Cherian, G. (1994). Protective effects of metallothionein on streptozotocin induced diabetes in rats. Life Sciences, 55: 43-51.

Zalewski, P. Millard, S. Forbes, I. Kapaniris, O. Slavotinek, S. Betts, W. Ward, A. Lincoln, S. and Mahadevan, I. (1994). Video image analysis of labile $\mathrm{Zn}$ in viable pancreatic islet cells using specific fluorescent probe for Zn. J Histochem. Cytochem., 42: 877-884.

Zimny, S. Gogolin, F. Abel, J. and Gleichmann, H. (1993). Metallothionein in isolated pancreatic islet cells of mice: induction by zinc and streptozotocin, a naturally occurring diabetogen. Arch. Toxicol, 67: 61-65. 